\title{
Atmospheric Prediction Models
}

\author{
D. Burridge, Reading and A. Wiin-Nielsen, Geneva \\ (Respectively : European Centre for Medium Range Weather Forecasts ; Secretary-General, World Meterological Organization)
}

The first numerical integration of a simple atmospheric non-linear model based on the barotropic vorticity equation and using atmospheric data was carried out about 30 years ago by Charney, Fjørtoft and von Neumann. Since then, there has been a very rapid development in the design of atmospheric models which gradually have become more realistic by including a description of those physical processes of importance in the behaviour of the atmosphere. An equally significant development has taken place in the design of the procedures used for the numerical integration of the complicated equations that describe the large scale atmospheric dynamics and thermo-dynamics. At the same time, larger and faster computers have allowed the meteorologists to incorporate more physical processes and better numerical methods into their models.

\section{Mathematical and Physical Basis for Numerical Models}

\section{Exact Equations}

The physical laws governing the atmosphere are the gas law, the laws of conservation of mass and "dissolved" substances (such as water vapour), the momentum equation and the first law of thermodynamics. The physical quantities entering these laws are the three dimensional velocity vector $\mathbf{v}$ relative to the earth, the temperature $T$, the pressure $p$, the density of air $\varrho_{d}$ and the density of the water vapour $\varrho_{w}$. Some atmospheric models have also conservation laws for other constitutents such as liquid water (cloud water) and ozone. As some of these physical laws contain sources and sinks it is necessary to provide a second set of expressions which specify the sources in terms of known quantities and the physical quantities entering the laws. It is thus required to describe the heating and cooling of the atmosphere by specifying the radiative transfer, the turbulent transfer of heat, the thermodynamic effects of evaporation (sublimation) and condensation and the formation of precipitation. Similarly, the turbulent transfer of momentum and the viscous dissipation (F) must be specified to complete the momentum equation. For the dissolved substances turbulent transfer must also be described.
The basic equations, relative to a frame rotating with the earth's angular velocity $\Omega$, and denoting the position vector from the centre of gravity of the earth by $\mathbf{R}$, are :

$$
\begin{gathered}
d \varrho_{d} / d t=-\varrho_{d} g r a d \cdot \mathbf{v} \\
\text { (conservation of mass, dry air) } \\
d \varrho_{w} / d t=-\varrho_{w} g r a d \cdot \mathbf{v}+S \\
\text { (conservation of water vapour) } \\
\mathrm{C}_{\mathrm{p}, \mathrm{a}} d T / d t-\beta\left(T / \varrho_{\mathrm{a}} \text { ) } d p / d \mathrm{t}=Q\right. \\
\text { (first law of thermodynamics) } \\
\quad p=\varrho_{a} R_{a} T \\
\text { (gas equation) } \\
d \mathbf{v} / d t=\operatorname{grad} p / \varrho_{\mathrm{a}}-\operatorname{grad}(\mathrm{gz}) \\
-\Omega \times \Omega \times \mathbf{R}-2 \Omega \times \mathbf{v}+\mathbf{F} \\
\text { (momentum) }
\end{gathered}
$$

where

$$
\mathrm{e}_{\mathrm{a}}=\mathrm{e}_{\mathrm{d}}+\mathrm{e}_{\mathrm{w}}
$$

\[ \varrho_{a}=e_{d}+\varrho_{w} \]
A table of symbols is given opposite.

For the models that include moisture (or other dissolved substances) it is assumed that the atmosphere consists of a mixture of perfect gases leading to the form (4) of the gas equation in which $R_{\mathrm{a}}$ is a suitably defined gas constant for the mixture. (A similar expression is required for $\left.c_{p, \mathrm{a}}\right)$. We note further that $\beta$, the coefficient of thermal expansion, is given by

$$
\beta=\varrho_{\mathrm{a}} \circlearrowright \varrho_{\mathrm{a}}^{-1 / \partial T}=\varrho_{\mathrm{a}} R_{\mathrm{a}} / T
$$

The underlying assumptions permitting the above formulation of the physical laws are basically the continuum hypothesis for the atmosphere, and thermodynamic equilibrium. When the state of the atmosphere is altered by an irreversible process, $Q$ in (3) will contain the heating which would occur in a reversible process giving rise to a similar change. Strictly, this precludes the application of these equations to very high atmospheric levels where these assumptions are invalid.

The diabatic terms, $F, Q$ and $S$, which contain the contribution from the irreversible processes are discussed later.

The models described here have, to our knowledge, not been used for operational forecasting.

\section{Approximate Forms}

of the Governing Equations

The exact equations set out above describe all types and scales of atmospheric phenomena. For prediction purposes, we can only resolve the scale of motion which can be analysed with the standard data, and it has become common practice to simplify the equations by omitting small terms, in this way filtering out unwanted motions. The approximate equations are usually derived by considering the temporal and spatial scales of interest. For large scale, middle latitude atmospheric conditions, the following scales for the dependent variables are observed:

$$
\begin{array}{rlrl}
U & \sim 10 \mathrm{~ms}^{-1} & & \text { (horizontal velocity) } \\
W & \sim 10^{-2} \mathrm{~ms}^{-1} & \text { (vertical velocity) } \\
L & \sim 10^{6} \mathrm{~m} & & \text { (horizontal length) } \\
H & \sim 10^{4} \mathrm{~m} & & \text { (vertical depth) } \\
\Delta p & \sim 10^{3} \mathrm{Nm}^{-2} & & \text { (horizontal pressure) } \\
L / U & \sim 10^{5} \mathrm{~s} & & \text { (advective time) }
\end{array}
$$

Where these scales are used to analyse the vertical equation of motion in (5) it is found to a very good approximation that the vertical pressure is balanced by the gravitational force:

$$
-\partial p \partial z-g \varrho_{a}=0
$$

The hydrostatic equation (8) leaves us without a prognostic equation for $W$, the vertical velocity, but adopting (8) $W$ can be determined diagnostically from the remaining equations. (1), (2), (3) and (4) can be combined to derive an equation for $\delta p / \partial t$ and a second independent equation for $\partial p / \partial t$ can be obtained from (8). Eliminating $\partial p / \partial t$ one obtains a single equation from which $W$ can be determined from the horizontal velocities and the other parameters in the model. Since $W$ is determined diagnostically using (8) it is no longer an "observable" quantity, but the vertical velocity used in the model is one which requires that hydrostatic equilibrium is maintained everywhere and at all times. An additional consequence of $(8)$ is that other approximations must be made to secure that the resulting system has reasonable conservation laws for energy (in the absence of sources and sinks) and for momentum. These approximations amount to excluding $W$ from the definition of kinetic energy and replacing the radial distance $R$ by the radius of the earth $a$. This is justified because the atmosphere is a fairly thin envelope surrounding the 


\section{TABLE OF SYMBOLS}

$=(u, v, w)$ - three dimensional velocity vector relative to the earth

$T=$ temperature

$p \quad=$ pressure

$\rho_{\mathrm{d}}=$ density of dry air

$\rho_{\mathrm{w}} \quad=$ density of water vapour

$\rho_{\mathrm{a}} \quad=$ density of moist air

F $\quad=$ viscous dissipation for momentum

= water vapour source/sink term

Q $\quad=$ diabatic heating

$R_{\mathrm{a}}=$ gas "constant" for moist air

$c_{p, a}=$ specific heat at constant pressure for moist air

$\beta=$ coefficient of thermal expansion for moist air

$g \quad=$ acceleration due to gravity

$\Omega \quad=$ earth's angular velocity

$x, y, z=$ spatial co-ordinates

$\mathbf{R} \quad=$ radial co-ordinate measured from the centre of the earth

$t=$ time

$d / d t=\partial / \partial t+\mathbf{v} \cdot \operatorname{grad}$

$\operatorname{grad} h=(\partial / \partial x, \delta / \partial y, 0)$ horizontal gradient operator

earth. The resulting equations are called the "primitive equations" by the meteorological community.

One of the most important consequences arising from the adoption of ( 8 ) is the removal of vertical travelling sound waves whose presence sets severe restrictions on the numerical integration schemes which are used to determine the evolution in time of the model variables. The existence of these waves in the "exact" equations severely restricts the time-step that can be taken with explicit timestepping schemes since the vertical grid length in numerical models is usually of the order of one $\mathrm{km}$. With the speed of sound being about $300 \mathrm{~ms}^{-1}$ this would restrict the time step to $10^{3} \mathrm{~m} / 300 \mathrm{~ms}^{-1}=\sim 3 \mathrm{~s}$. In fact, numerical models based on the "exact" equations are now being developed to study non-hydrostatic effects in small-scale systems. Special techniques enable longer time-steps to be employed.

The first models for forecasting the weather used even more approximate forms of the "exact" equations, the so-called quasi-geostrophic system. Derived by a scale analysis, mainly for the horizontal momentum equations, the geostropic wind

$$
-\operatorname{grad}_{h} p / \varrho_{a}-2 \Omega \times \mathbf{v}=0
$$

had to serve for advection purposes.

Although quasi-geostrophic models still find a place in some national meteorological forecasting services, the trend is to reserve the more ap- proximate models for analytical studies where it is sometimes possible to obtain closed form solutions in terms of elementary functions. These theoretical approaches do, in many cases, give considerable insight into the dynamics of special phenomena.

The primitive equations are highly non-linear and the only known methods of solution are numerical. The finite difference approach replaces the continuous partial differential equations by difference equations, whose solutions give the values of the dependent variables on a three dimensional grid or lattice of points. Other schemes for numerical solutions involve expanding the dependent variables in a finite series of orthogonal functions which are then substituted into the continuous equations to give evolutionary equations for the amplitudes associated with the individual functions. A $100 \mathrm{~km}$ horizontal spacing between grid points is considered to be a fine mesh for operational weather forecasting. Vertical grid lengths of $1-2 \mathrm{~km}$ are typical for models covering the lowest 15 to 20 $\mathrm{km}$ of the atmosphere.

\section{Physical parameterisation}

The important physical effects to include, i.e. the various component parts of the source/sink term F, $Q$ and $S$, are the interactions of the atmosphere with the surface involving momentum, heat and moisture transfers, energy balance at the earth's surface, topographic effects, radiative heating, cumulus convection and large scale condensation. Most of these physical effects occur on spatial scales which are considerably shorter than the grid lengths of most numerical models but their importance as the sources and sinks of energy and momentum for the atmosphere is obvious. These atmosphere processes are as such, sub-grid scale and cannot be described explicitly in the numerical calculations but can only be represented statistically or parametrically. The phrase "physical parameterization" has been used by meteorologists when referring to this part of numerical models of the atmosphere.

The major sink for momentum and sources of heat and moisture is the earth's surface. Vertical fluxes of heat are greatest in a thin layer near the ground, the planetary boundary layer (PBL). The effect of the PBL on the rest of the atmosphere rather than the details of the boundary layer itself is required in most numerical models. If we regard the grid point values, $u$, of the horizontal velocity as a mean value for the grid square surrounding these points then within the grid square the velocity is $u+u^{\prime}$ where $u^{\prime}$ is a perturbation from the mean. This leads to Reynolds stress terms or correlations, such as $\overline{u^{\prime} w^{\prime}}$ and $\overline{w^{\prime} T^{\prime}}$ which are the vertical fluxes of momentum and heat by sub-grid scale processes. Simple models parameterize these terms with bulk aero-dynamic formulae using drag coefficients depending on the underlying surface, and vertical diffusion schemes. These are first order closure schemes. In other more sophisticated models, prognostic equations are derived for $\overrightarrow{u^{\prime} w}$ in terms of the mean flow quantities and higher correlations, with assumptions about these correlations (closure assumptions). These high order closure schemes have received very little testing on account of their complexity and expense.

When large quantities of heat and moisture are supplied to the lower layers of the atmosphere (this occurs in the tropics and frequently when cold air moves over a warm sea in extra tropical latitudes) the atmosphere tends to become de-stabilized leading to convective storms which transport heat, moisture and momentum vertically into the atmosphere. The horizontal scale of these storms is of the order of only a few kilometres but they are an important transport mechanism in the atmosphere. Again parameterization is required, usually relating vertical fluxes to the stability of the atmosphere and the rate of access of energy and moisture into a grid square.

The effects of the formation of evaporation and precipitation have also to be included in these numerical models and this is usually accomplished in forecasting models with little or no sophisticated cloud physics. In most models the parameterization amounts to deciding whether a small volume of the model atmosphere is supersaturated or not. If the volume is supersaturated sufficient precipitation is produced to maintain saturation. This process releases latent heat which modifies the temperature field.

In most operational short range forecasting models there are no calculations of the direct effect of the sun's radiation except perhaps to compute the energy balance near the surface of the earth. This is easily understood, first because it is a slow acting process whose inclusion has little effect on short-range predictions of the weather, and second because radiation calculations are notoriously expensive and complicated. The sun's radiation and the long wavelength 
radiation from the earth are affected by the presence of clouds, water vapour, carbon dioxide, ozone, aerosols, etc. It is highly unlikely that any weather prediction model will attempt anything like an exact calculation and at best it will be gross parameterization. The most sophisticated schemes consider the effects of water vapour, clouds, ozone and carbon dioxide. The current approach is to derive a parameterization from detailed calculations based on the latest experimental data and theory by some fitting procedure. Even if this is a reliable approach, the importance of forecasting the cloud cover and depth (another sub-grid scale effect) cannot be over-emphasized.

\section{Numerical Aspects}

The first decision to be taken is the choice of the area of integration and the lateral boundary conditions. Boundary conditions at the surface of the earth and the "top" of a model are set by physical and kinematic constraints, whereas those at lateral boundaries are in some sense arbitrary. For global models, no problem arises since all that is required is continuity and regularity of the solution. For open boundaries, the primitive equations are ill-posed and this problem is overcome by the addition of smoothing terms near the boundaries. The exact equations are, in fact, well posed and stable lateral boundary conditions can be formulated for them. For economy, many national weather services use limited area models covering the areas of the globe in which they are interested. The boundary values are either maintained constant in time or are derived from forecasts for much larger areas produced with simpler or coarser mesh models.

Numerical integration of the models described in the last section is usually accomplished using grid point schemes in which the values of the dependent variables are defined on a three dimensional grid of points. Experience and simple theoretical arguments indicate that the aspect ratio of the grid lengths should be about $10^{-2}$, in order to model consistently the dynamics of developing storms whose horizontal scales are of the order of $10^{3} \mathrm{~km}$. Typically the most recently developed models have between 10 and 20 grid points in the vertical.

The use of high order difference formulae is not common since the experience with meteorological models is that they provide very little extra accuracy over the simple centred second order difference formulae.
Other deficiencies appear to be more important than finite difference errors provided the horizontal mesh is reasonably fine, less than $300 \mathrm{~km}$ say.

Whatever description is used for the spatial representation the model equations reduce to the following form

$$
\partial x / \partial t+\mathbf{M}(x)=0
$$

where $x$ is a vector representing all the model's dependent variables whether they are grid point values, coefficients of functions in a series representation or nodal values in a finite element scheme. The function $\mathbf{M}$ may be written

$M(x)=L(x)+N(x)+D(x)$ (11) where $L$ is a linear operator, $N$ and $D$ non-linear operators representing the non-linear adiabatic and diabatic terms, respectively. Many techniques for the integration of (10) in time are in use such as explicit schemes, splitting schemes in which the individual parts of $\mathbf{F}$ are treated independently as if the others didn't exist, and semi-implicit schemes. The system has, as solutions, rapidly horizontally moving gravity waves, the fastest of which move at $300 \mathrm{~ms}^{-1}$. These waves give rise to stability criteria restricting the length of the time-steps, $\Delta t$, that can be used in any explicit numerical integration scheme. Typically it is found that we require

$$
\Delta t<\Delta x /\left(C+U_{\max }\right) 2^{1 / 2}
$$

where $C=300 \mathrm{~ms}^{-1}$ is the speed of the gravity waves and $U_{\max }$ the maximum horizontal wind speed. For $\Delta x=$ $100 \mathrm{~km}, U_{\max }=100 \mathrm{~ms}^{-1}$ (horizontal wind speeds of $50 \mathrm{~ms}^{-1}$ and higher are frequently encountered in the stratosphere above $25 \mathrm{~km}$ ) which allows maximum time-steps of 180 seconds. In the first numerical models these waves were filtered out and the stability restrictions depended only on the wind speed. In the atmosphere, very little energy is contained in gravity wave modes but their presence is maintained because dispersion of gravity waves on a rotating sphere is the mechanism by which the coriolis force and horizontal pressure gradient are maintained in approximate balance Fully implicit schemes, such as the Crank Nicholson scheme, set no restriction on the time-step that can be used for (10), but this would necessitate the solution of a complicated non-linear equation at each time-step. Another approach is the use of a partially implicit or semi-implicit scheme in which only the linear terms are treated implicity. In most meteorological problems this amounts to solving a 3-dimensional Poisson equation, which can be accomplished by separation of variables and the use of
Fourier transforms and tri-diagonal matrix inversions for grid point and finite element models.

In addition to satisfying linear stability criteria, numerical models must have long term stability. The possibility and occurrence of other types of computational instability of numerical schemes arises because of the nonlinearity of advection processes. When two fields are multiplied together, the interactions involving the shortest waves cannot be correctly represented, as these interactions produce scales which are too fine to be resolved on the original grid and are instead misinterpreted as contributions towards longer scales. These spurious interactions are usually called aliasing errors and can lead to instability. In order to avoid the worst effects of this process many models use artificial dissipation and smoothing to damp out or remove the short scales, Another approach is to design finite difference schemes which conserve non-linear quantities such as energy. These conservative schemes are not only important from a physical point of view but also from a numerical one by inhibiting the development of nonlinear instability. Spectral and finite element models which are usually based on a Galerkin approach, in which the continuous equations are satisfied in a least-squares sense, are

(a) Three-dimensional Fields

$T \quad$ - temperature

$u, v \quad$ - horizontal components of velocity

$q=\varrho_{\mathrm{w}} / \varrho_{\mathrm{a}}-$ humidity mixing ratio

$\Delta T_{\mathrm{R}} \quad-$ radiation heating/

and cooling

(b) Two-dimensional Fields

$\begin{array}{ll}H_{S} & - \text { topography } \\ p_{S} & - \text { surface pressure } \\ T_{S} & - \text { surface temperature } \\ P_{\mathrm{R}} & - \text { large scale accumula- } \\ & \text { ted rain } \\ p_{\mathrm{C}} & - \text { sub-grid scale } \\ \partial P_{R} / \partial t & \text { convective rain } \\ \partial P_{C} / \partial t & \text { - rainfall rate } \\ \Delta T_{S} & - \text { rainfall rate } \\ \Delta q_{S} & \text { - surface exchange } \\ & \text { of heat } \\ & \text { of moisture }\end{array}$

surface parameters, climatic type indicators, constants for vertical and horizontal diffusion. 
usually free from aliasing errors which would lead to this type of instability.

To conclude this section we shall describe the characteristics of a global grid point model being prepared for operational forecasting at E.C.M.W.F. This model probably represents a "state of the art" model for weather forecasting.

The model has 15 horizontal levels (15 grid points between the ground and the top) and has 28,800 points on each horizontal surface. The model's variables are shown in the list on the opposite page.

A three-dimensional field for radiation heating is necessary because the radiation field will not be computed every time-step in view of the heavy computational expense involved. Other variables are also required, such as the density and vertical velocity but these are diagnosed from other variables when required. The integration scheme is a three level scheme. With extra "work space" for the integration scheme, seven 3dimensional fields are required to define the state of the model at a single time level. In order to perform the time-stepping algorithm, two time levels of data are necessary. This amounts to $2 \times 7 \times 15$ (number levels $\times 28,800$ (grid points on each level) $=6$ million numbers.

The CRAY-1 computer at E.C.M.W.F. has space for at most 1 million numbers. A conventional way of organising this type of computation is to divide it into a large number of records which are stored on a computer disc ; a convenient division for our global model, which uses latitude and longitude as horizontal co-ordinates, is to place all the data for a single line of latitude in one record. These records are read and written sequentially from the disc to and from the computer's memory. Sufficient data is retained in the computer to perform the computions for a single line of latitude (a vertical slice). The computer program and hardware is arranged in such a way that the reading, writing and computing takes place asynchronously. With this organization 280,000 numbers are kept in the computer at any one time. Spectral models and finite element models can be organized in a similar fashion.

For this model, it takes five hours to produce a 10 day forecast on the CRAY-1 computer, estimated to be 10 times faster than with an IBM $360-195$. The whole model is in the FORTRAN programming language and could, with optimization (machine language programming), be speeded up by a factor of two.

\title{
POSTDOCTORAL POSITION IN SOLID STATE PHYSICS WITH POSITIVE MUONS ETH ZURICH / SWITZERLAND
}

An open postdoctoral position has become available in the group for Muon Spin Rotation ( $\mu$ SR) Spectroscopy at the

Laboratory for High Energy Physics of ETH Zurich (LHE)

The research programme of this group involves the study of magnetic and electronic properties of metals, metal alloys and metal hydrides.

An experimental physicist (Ph.D.) is sought, who is particularly inclined towards experimental work and who is willing to work in a team of five to eight physicists. A solid state background would be preferable but is not a prerequisite. The interdisciplinary character of the $\mu$ SR-research requires a liking in using and handling quite different equipment and measuring tasks (modern fast counting electronics, on-line computing, precision measurements of magnetic fields (NMR), cryostats, ovens, temperature measurements, physical characterization of samples).

Interested persons are asked to contact as soon as possible :

\author{
Dr. A. Schenk, LHE \\ c/o SIN, CH - 5234 Villigen, Switzerland
}

Trends in Computational Meteorology

In the past 20 years a great deal of progress has been made in short range forecasting with numerical models based on the equations described in the second section. For this, an accurate knowledge of the initial state and an accurate treatment of the adiabatic effects (the advection etc.) is required. Short range forecasting models usually have particularly simple physical parameterizations and neglect the slow acting physical processes. At the other end of the spectrum, more sophisticated numerical models, which take into account or have a representation for, all the important physical processes, have been used to simulate the earth's climate. The European Centre for Medium Range Weather Forecasts has perhaps a more difficult problem, forecasting the weather for 10 days ahead, since both the initial data and the physics are important in order to describe correctly the initial detail and evolution of the weather and to enable the model to predict the modification and decay of these systems and to predict the birth of new disturbances.

There is a great body of evidence to suggest that short range weather forecasting will improve dramatically through an improvement in the specification of the initial state of the atmosphere rather than the models alone. For climate simulations, the models are obviously deficient. The First Garp Global Experiment (F.G.G.E.) which began in late 1978 and continued through 1979 , is an attempt to provide a solution to both problems. During that year the earth's atmos- phere was observed more intensely than it had ever been before. These observations will form a basis for evaluating short range forecasting models and for improving our knowledge of the physics of the atmosphere, which will in turn hopefully improve parameterization schemes for large scale models. This research and development will involve building special models to simulate the boundary layer, convective storms and other phenomena which cannot be represented explicitly in large global models. Such work involves an enormous amount of computing capacity and facilities. Higher speed machines are needed to perform the numerical experiments, sophisticated data processing systems to manage the data bases of observations, analyses and forecasts, and to present the results in a usable form.

\section{SUGGESTED READING}

For the interested reader the following publications give much more information than we could provide in this short review.

"Introduction to Theoretical Meteorology" by S.L. Hess (Holt, New York) 1959 (standard elementary text book on meteorology).

"An Introduction to Dynamic Meteorology" by J.R. Holton (Academic Press, New York and London) 1972 - (standard elementary text book on meteorology).

"Dynamic Meteorology" edited by P. Morel (D. Reidel Pub. Co.) 1973.

"The Physics of Clouds" by B.J. Mason 2nd Ed. (O.U.P.) 1971.

"Radiation in the Atmosphere" by K. Ya. Kondratyer International Geophysics Series Volume 12 (Academic Press) 1969.

"The Nature and Theory of the General Circulation of the Atmosphere" by E.N. Lorenz (World Meteorological Organization, Geneva) 1967. 